\title{
A Rare Variety of Adenoid Cystic Carcinoma involving Submandibular Salivary Gland
}

\author{
Sanjoy Kumar Ghosh, ${ }^{1}$ Saikat Samaddar, ${ }^{1}$ Swagatam Banerjee, ${ }^{1}$ Diptanshu Mukherjee ${ }^{1}$
}

\section{Introduction}

\section{ABSTRACT}

Adenoid cystic carcinoma, an uncommon malignant salivary gland neoplasm, is known for its long clinical course, indolent growth, neural invasion, local recurrence and late distant metastasis. Three distinct histological patterns have been identified, amongst which, solid pattern is the rarest one having the worst prognosis.

\section{Materials And Methods}

A case of sixty year old male patient presented with a swelling in the left side of the neck, below the jaw gradually enlarging during the last three years. FNAC from the neck swelling was suggestive of benign adenoma of the salivary gland, possibly basal cell adenoma or pleomorphic adenoma. Submandibular gland was excised. Histopathological examination confirmed the solid pattern of adenoid cystic carcinoma, which is the rarest among the three distinct histological subtypes of the malignancy. Discussion

Adenoid cystic carcinoma is well known for its perineural spread. In cases of submandibular gland adenoid cystic carcinoma, surgical wide excision is very much possible and in T1 or T2 cases post-operative radiotherapy may not be needed. In our case, post operative radiotherapy was given for best local control, as the tumour showed histopathlogical solid variety, grade III pattern, which is known to have the worst prognosis.

Keywords

Carcinoma, Adenoid Cystic; Submandibular Gland Neoplasms

A denoid cystic carcinoma, first described as "cylindroma" by Billroth in 1859, accounts for $10 \%$ of all salivary neoplasm and about $30 \%$ of all malignancies of the major and minor salivary glands, palate being its most common location in the oral cavity. ${ }^{1,2}$ These tumours are usually very slow growing mass with an encouraging five year survival rate, but late distant metastasis with local recurrence accounts for rather low long term survival rate. ${ }^{3}$

Adenoid cystic carcinoma is very much unique for two reasons. First, it has a marked propensity for neural invasion, which occurs up to $50 \%$ of cases, causing pain (the most common symptom) and the second being its protracted natural history. Patients can even survive for 10-15 years even when local recurrence or distant metastasis has occurred. ${ }^{3}$ Pulmonary metastasis is the commonest. Three histological patterns of adenoid cystic carcinoma are recognised: (1) Cribriform or Swisscheese pattern which is the most common; (2) Tubular, the next most common; (3) Solid, which occurs in around 21 percent of cases, and has the worst prognosis. ${ }^{1,4,5}$

Aggressive surgical resection is the mainstay of treatment. Post operative radiotherapy has been advocated to reduce local recurrence. Some consider, this should be standard therapy for adenoid cystic carcinoma of the head and neck and preferentially for high risk patients. ${ }^{6}$ However, another study considered radiotherapy to be unnecessary where surgical margins were negative, ${ }^{7}$ neutron beam therapy may be more effective. ${ }^{8,9}$ Chemotherapy has not produced good results. ${ }^{10}$

\section{Case Report}

A sixty year old male patient was referred to

1 - Department of ENT, Medical College, Kolkata

\section{Corresponding author:}

Dr Sanjoy Kumar Ghosh

email: sanjoyghoshcmc007@gmail.com 
Otorhinolaryngology Clinic with a swelling in the left side of the neck below the jaw for three years. The swelling was insidious in onset and gradually progressive to attain the size of a large marble. There was no associated pain and discharge from the swelling or difficulty in deglutition. The swelling did not increase in size during meals. The systemic examination was not contributory. Head and neck examination revealed $3 \times 3 \mathrm{~cm}$ globular swelling on the left submandibular region (Fig. 1). Overlying skin had no scar or sinus. Local temperature was not raised and the swelling was non tender. The consistency was firm to hard, mobile, and not fixed to the overlying skin or any underlying structure. The swelling was bimanually palpable. The neck was supple with no significant lymphadenopathy. No bruit, thrill or venous engorgement was detected.

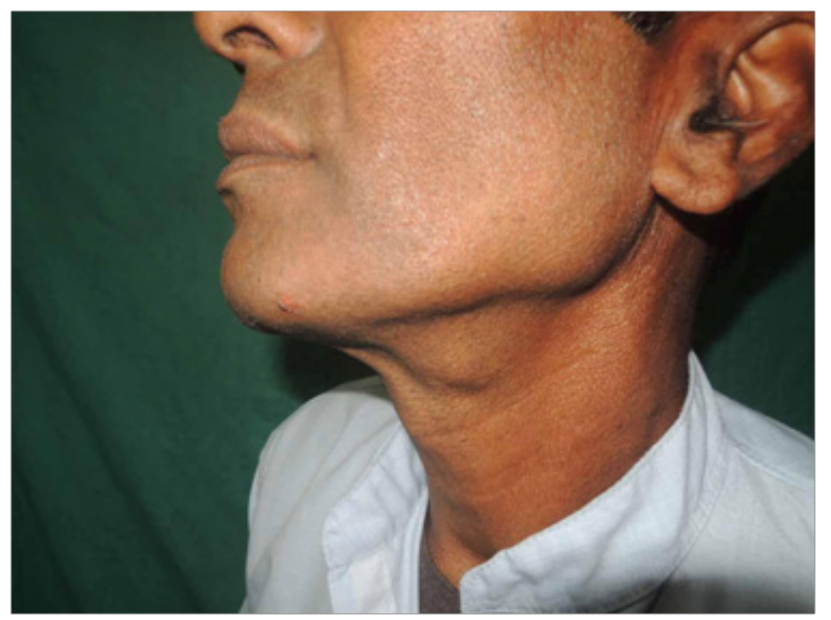

Fig. 1 Lateral view of the left side of neck showing swelling in left submandibular gland

X-ray neck showed no significant abnormality. CT scan of the neck showed a homogeneous mass lesion within left submandibular gland without any lymphadenopathy. FNAC from the neck swelling was suggestive of benign adenoma of the salivary gland, possibly basal cell adenoma or pleomorphic adenoma.

Patient was operated under general anaesthesia to excise the left upper neck mass, located at submandibular region. The submandibular gland was bulky and a hard swelling was found in the lower portion of the gland. The gland had no adhesion to the surrounding structures and the capsule was intact (Fig. 2). The submandibular

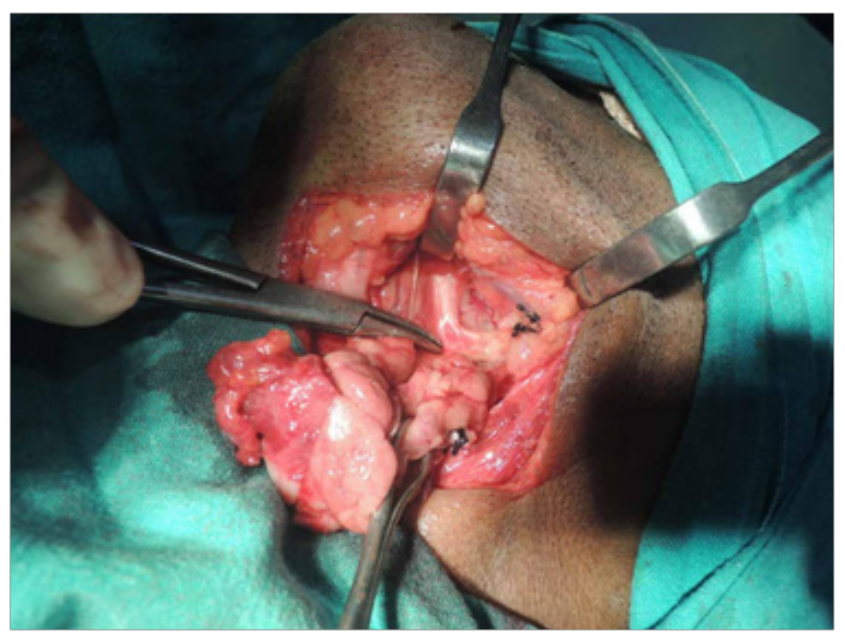

Fig. 2 Excision of submandibular gland - dissection from loop of lingual nerve

gland was removed completely along with the duct and sent for histological examination.

On gross examination the specimen was partially firm and partially hard, yellowish white in colour, measuring $4 \times 2.5 \times 1 \mathrm{~cm}$. On microscopic examination nests of basaloid cells with hyperchromatic nuclei and scanty neoplasm was found. The cells did not show significant pleomorphism. The nests were separated by dense stroma. Occasionally the cells were arranged in gland like spaces containing hyaline material within (Fig. 3). Perineural invasion was noted. Section from the

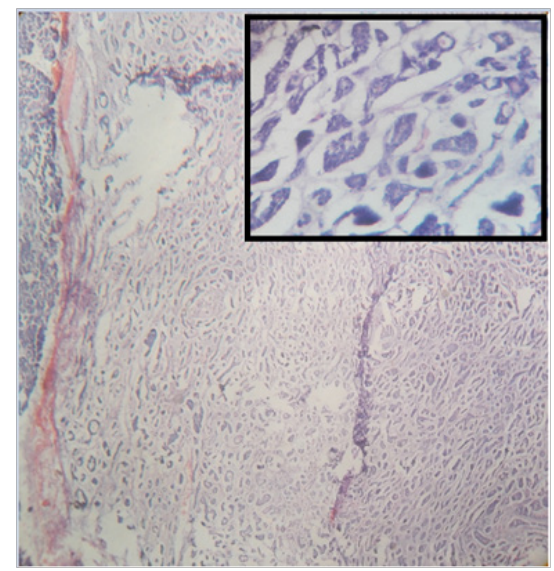

Fig. 3 Hematoxylin and Eosin stained HP section (10x) of Adenoid cystic carcinoma - solid pattern (inset showing view of hypermagnification- 100x) 
apparently normal area showed unremarkable salivary gland tissue. So it was confirmed to be an Adenoid cystic carcinoma - solid pattern, grade III, pT1N0M0.

\section{Discussion}

Adenoid cystic carcinoma is a slow growing but highly malignant neoplasm of the salivary gland, commonly affecting the minor salivary glands of head and neck region. ${ }^{1,2}$ Minor salivary glands are affected most by adenoid cystic carcinoma $(40 \%)$, followed by the submandibular gland (18.2\%) and the parotid gland $(17.3 \%) .{ }^{11}$

The solid histological pattern is seen only in 21 percent of cases. The solid pattern of adenoid cystic carcinoma, involving submandibular salivary gland, presented here, was a very rare case. Most individuals diagnosed with this disease are in their fourth decade of life, though a wide age range has been reported. The female and male ratio is approximately $3: 2$. Usual presentation is an asymptomatic slow growing mass, the absence of symptoms being responsible for late diagnosis. Here our patient was sixty years old male patient having a slow growing mass in left sided submandibular region without other symptoms.

The three major histological patterns of growth have been described: cribriform, tubular and solid. Combinations of the patterns are common. The prognosis of adenoid cystic carcinoma is greatly influenced by the pattern of growth, tubular pattern having the best prognosis while solid is known to have the worst prognosis. ${ }^{1,4,5}$

Adenoid cystic carcinoma has a relatively slow growth and infrequent lymph node metastasis but is well known for its perineural spread. In a study of 160 patients of adenoid cystic carcinoma by Fordice et al, disease specific survival was $89 \%$ at five years but only $40 \%$ at fifteen years. ${ }^{5}$ Distant metastasis was the commonest cause of treatment failure. Lung is the most common site for distant metastasis, followed by liver. Spiro in his retrospective study of 196 patients followed up for 10 years reported some form of treatment failure in $68 \%$, distant metastasis in $38 \%$, and lung involvement either alone or in addition to other sites in $34 \% .{ }^{12}$ In cases of submandibular gland adenoid cystic carcinoma, surgical wide excision is very much possible and in $\mathrm{T} 1$ or $\mathrm{T} 2$ cases post operative radiotherapy may not be needed. ${ }^{7}$ Elective neck dissection is usually not done in N0 cases as lymph node metastasis is not common at all. Some authors prefer radiotherapy as a standard mode of treatment and especially in high risk cases. ${ }^{6}$

The involved submandibular gland, in the present report, was not adherent to surrounding structures and the surgical margin was clear. Lingual and hypoglossal nerves were free of disease and no lymph node was involved in surgical field. Histopathological examination of excised submandibular gland revealed adenoid cystic carcinoma - solid pattern with perineural invasion, grade III, pT1N0M0. After complete surgical removal patient was sent to radiotherapy clinic for radiotherapy for best local control as the tumour was solid variety grade III pattern of adenoid cystic carcinoma which has most poor prognosis. ${ }^{6}$ Patient is under follow up for last 6 months having no local or systemic complaint till now.

\section{References}

1. Chummun S, McLean NR, Kelly CG, et al. Adenoid cystic carcinoma of the head and neck. Br J Plast Surg.2001; 54:476-80

2. Khan AJ, DiGiovanna MP, Ross DA, et al. Adenoid cystic carcinoma: a retrospective clinical review. Int J Cancer. 2001; 96:149-58

3. Westra WH: The surgical pathology of salivary gland neoplasms. Otolaryngol Clin North Am 1999; 39: 919-43

4. Nascimento AG, Amaral AL, Prado LA, Kligerman J, Silveira TR. Adenoid cystic carcinoma of salivary glands. A Adenoid cystic carcinoma of salivary glands. A study of 61 cases with clinicopathologic correlation. Cancer. 1986; 57:312-9

5. Fordice J, Kershaw C, El-Naggar A, Goepfert H. Adenoid cystic carcinoma of the head and neck: predictors of morbidity and mortality. Arch Otolaryngol Head Neck Surg. 1999;125:149-52

6. Bradley PJ: Submandibular gland and the minor salivary gland neoplasms. Curr Opin Otolaryngol Head Neck Surg 1999; 7:72-8

7. Prokopakis EP, Snyderman CH, Hanna EY, et al.: Risk factors for local recurrence of adenoid cystic carcinoma: the role of postoperative radiation therapy. Am J Otolaryngol 1999; 20: 281-6

8. Prott F-J, Micke O, Haverkamp U, et al.: Results of fast neutron therapy of adenoid cystic carcinoma of the salivary glands. Anticancer Res 2000, 20: 3743-50

9. Douglas JG, Koh W-J, Austin-Seymour M, et al.: Treatment of 
salivary gland neoplasms with fast neutron radiotherapy. Arch Otolaryngol Head Neck Surg 2003, 129: 944-8

10. Terashima K, Shioyama Y, Nakamura K, et al; Long-term local control of recurrent adenoid cystic carcinoma in the parotid gland with radiotherapy and intraarterial infusion chemotherapy.; Radiat Med. 2006; 24:287-91
11. Bonaparte JP, Hart R, Trites J, Taylor MS. Incidence of adenoid cystic carcinoma in nova scotia: 30-year population-based epidemiologic study. J Otolaryngol Head Neck Surg. 2008; 37:642-8

12. Spiro RH. Distant metastasis in adenoid cystic carcinoma of salivary origin. Am J Surg. 1997;174:495-8 